\section{Full-field phase measure- ment by wavelength-tuning interferometry in the C-band}

\author{
Patrick Egan, ${ }^{a, b}$ Fereydoun Lakestani, ${ }^{a}$ \\ Maurice P. Whelan, ${ }^{a}$ and Michael J. Connelly ${ }^{b}$ \\ ${ }^{a}$ European Commission Joint Research Centre, Photonics \\ Group, Institute for Health and Consumer Protection, \\ Ispra, Varese, Italy \\ bUniversity of Limerick, Optical Communications Research \\ Group, Castletroy, County Limerick, Ireland \\ E-mail: patrick.egan@ul.ie
}

\begin{abstract}
Fluctuations in the output intensity and wavelength of an external cavity diode laser can introduce significant error to wavelength-tuned interferometric measurement. However, a robust phase-retrieval algorithm can compensate for these nonlinearities. Employing an inexpensive phosphor-coated charge-coupled device camera sensitive to C-band infrared, full-field interferometric phase retrieval utilizing wavelength tuning of a $1555 \mathrm{~nm}$ external cavity diode laser is reported. Phase measurement of a tilted mirror is presented with an estimated accuracy within $7 \mathrm{~nm}$. (๑) 2006 Society of Photo-Optical Instrumentation Engineers. [DOI: 10.1117/1.2401169]
\end{abstract}

Subject terms: infrared; phase retrieval; interferometry; phase measurement.

Paper 060498LR received Jun. 23, 2006; revised manuscript received Oct. 5, 2006; accepted for publication Oct. 6, 2006; published online Dec. 4, 2006.

Phase measurement techniques that employ movement in one arm of an interferometer are prone to errors caused by vibrations. With an unbalanced interferometer, scanning the wavelength of a tunable laser is equivalent to path length scanning with a fixed wavelength laser. Wavelength tuning inside the light source replaces external electromechanical scanning of the interferometer path length. Some advantages of this are that with an imaging device there are no moving parts in the interferometer, both rough and smooth surfaces can be profiled with the same setup, and the metrology is integral, ultraprecise, and always known (depending on laser quality). Wavelength-tuning interferometry has been used for noncontact millimeter surface topography ${ }^{1}$ and nanometer surface relief. ${ }^{2,3}$

Spurred by the telecom industry, there are some interesting devices at C-band wavelengths (1525 to $1565 \mathrm{~nm})$ that may prove useful for optical metrology: for example, distributed feedback lasers and semiconductor optical amplifiers. ${ }^{4}$ Furthermore, for some optical measurement applications, the higher refractive index of materials at longer wavelengths can be beneficial. ${ }^{5-7}$

Prohibitive to full-field metrology in the C-band, however, is the expense of imaging devices: for example, microbolometers, pyroview, and InGaAs cameras. In this work we investigate a phosphor-coated charge-coupled device (CCD) camera sensitive to $\mathrm{C}$-band infrared as an al-

0091-3286/2006/\$22.00 @ 2006 SPIE ternative. The camera is intended for beamwidth measurement and is up to 12 times cheaper than the prior devices. An external cavity diode laser provided functional yet distorted wavelength tuning (see Fig. 1), which introduced substantial phase error. One way to overcome these nonlinearities is to use analytic signal processing techniques to retrieve the interferometric phase.,

Previously, ${ }^{10}$ we developed an analytic signal phase retrieval algorithm used in a heterodyne interferometer with a white-light source. This work reports the adoption of the algorithm to wavelength-tuning phase retrieval with a simple measurement of a tilted mirror. Notable aspects of this work include the use of the inexpensive IR-CCD camera sensitive at $1555 \mathrm{~nm}$, and the effectiveness of the phase retrieval algorithm in the presence of wavelength tuning and camera nonlinearities.

The unbalanced Michelson interferometer of Fig. 2 was supplied $1.9 \mathrm{~mW}$ of light from an external cavity diode laser (2010 External Cavity Diode Laser, Environmental Optical Sensors Incorporated, Boulder, Colorado) centered at $1555.5 \mathrm{~nm}$. The laser was capable of tuning over a $78 \mathrm{~nm}$ range from 1521 to $1599 \mathrm{~nm}$. The light was not collimated; instead, the microlens was adjusted to yield a curved wavefront, preventing camera saturation. Without an objective lens, fringes were projected onto the camera focal plane. By ramp modulation of an internal piezoelec-
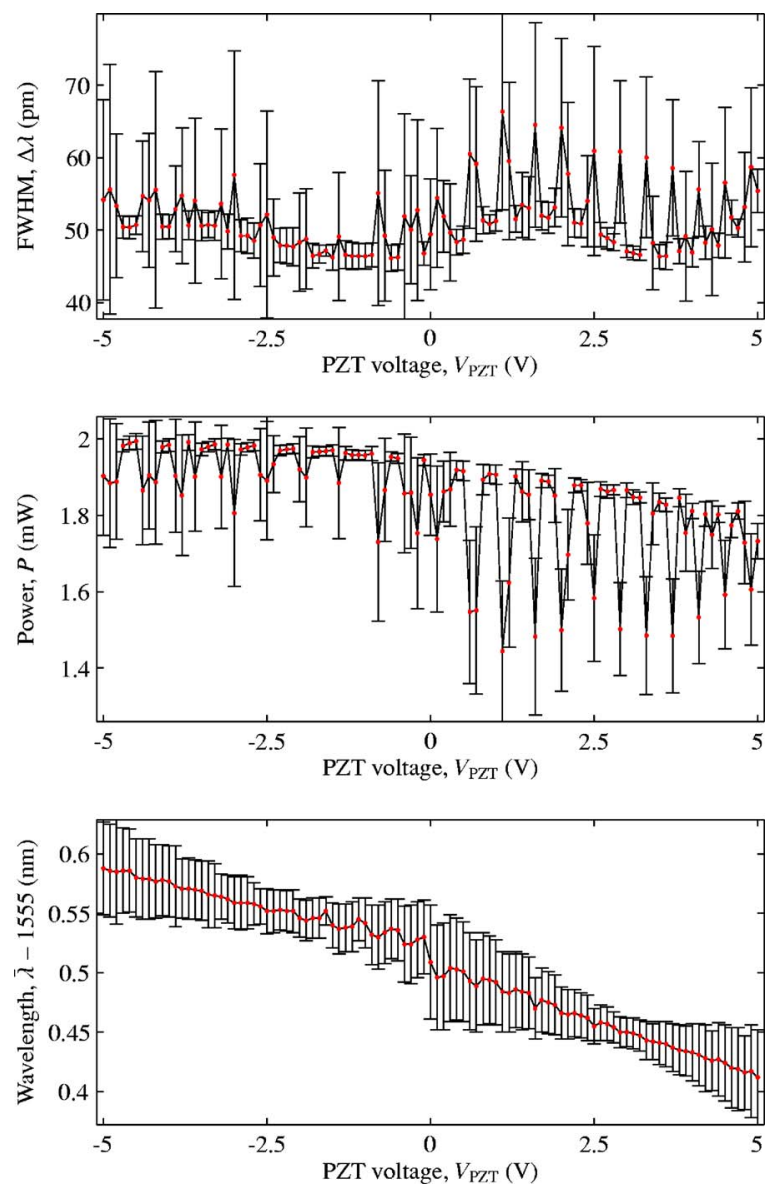

Fig. 1 PZT wavelength-tuning stability of external cavity diode laser monitored with an optical spectrum analyzer. Repeated ten times over $5 \mathrm{~h}$ with standard deviation error bars. 


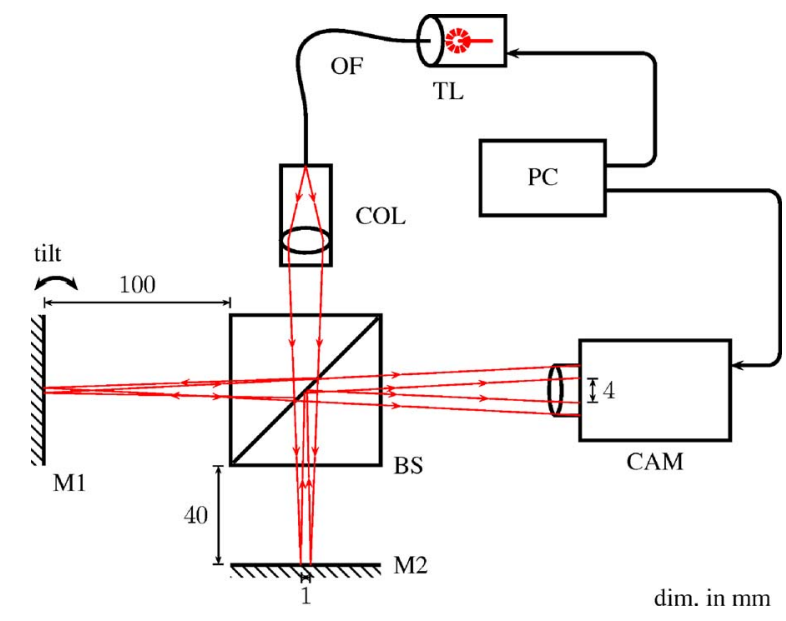

Fig. 2 Experimental setup. Components include: tunable laser (TL), optical fiber (OF), microlens collimator (COL), 50/50 beamsplitter (BS), mirrors (M1, M2), and phosphor-coated IR-CCD camera (CAM).

tric transducer (PZT), the wavelength of the laser was tuned over approximately $0.2 \mathrm{~nm}$, as in Fig. 1. This generated an interferometric cw signal of at least four periods. During the 4 s PZT ramp, 100 frames were captured with the camera. An external computer running LabVIEW controlled both PZT modulation and camera acquisition.

The IR-sensitive camera (SP-1550M, Spiricon Incorporated, Logan, Utah) consisted of a CCD focal plane with a phosphor coating that emitted visible radiation (antiStokes) when illuminated with infrared radiation in the range 1460 to $1625 \mathrm{~nm}$. However, the camera had a nonlinear response to light intensity: its primary design goal was for viewing IR emitting telecom devices and IR lasers in the C-band. The camera response to light intensity, both at C-band $(1555 \mathrm{~nm})$ and visible $(633 \mathrm{~nm})$ wavelengths, is shown in Fig. 3. Both responses are curve fitted with quadratic polynomials described by a squared correlation coefficient $r^{2}$. The different response behavior between C-band and visible wavelengths is likely due to the anti-Stokes process occurring in the $\mathrm{C}$-band, which is not as efficient as

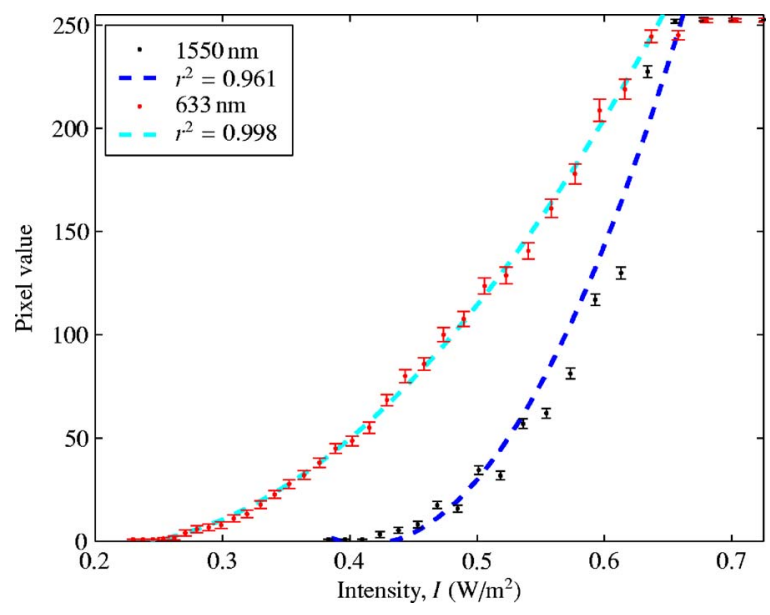

Fig. 3 Camera nonlinear response to light intensity at C-band and visible wavelengths. Error bars show standard deviation on five separate pixels with the measurement repeated five times. photon-electron conversion in the visible. Another disturbing characteristic of the camera is the very small intensity response range-not even $3 \mathrm{~dB}$ in the C-band. Although the dynamic range (signal-to-noise ratio) is close to $58 \mathrm{~dB}$ with only a $3 \mathrm{~dB}$ nonsaturable response range, interferometric fringes easily saturated and blackened the pixels. These nonideal effects were empirically observed through "fat" fringes (the camera is more sensitive to high light intensity than low) and easy saturation with pixel blooming and blotching effects, the latter due to phosphor-coating nonuniformities. Thus, combined with distorted wavelength tuning, complex system nonlinearities demand a robust algorithm to retrieve the interferometric phase.

The analytic signal phase retrieval algorithm was previously reported. ${ }^{10}$ It entailed building a window in the time domain equal to four periods of the interferometric cw signal. Our window of choice was a Hanning window convolved with itself in time: the Hann 2 window offered good extinction of a negative frequency component (at least $2.815 \times 10^{-5}$ at $f=-f_{c}$ ) and tolerated some carrier frequency variation. The window was multiplied by a factor $\exp \left(j 2 \pi f_{c} t\right)$ to center its spectral passband at the positive frequency of the carrier $f_{c}$. Convolving this window with the time history of each camera pixel yielded the analytic signal. The static phase difference between any two pixels was obtained by dividing the two analytic signals and taking the mean argument.

Despite wavelength tuning and camera response nonlinearities, a reasonable cw signal and spectrum was obtained, as shown in Fig. 4 with the Hann 2 window. The spectrum of the Hann 2 window allowed some fluctuation of the carrier frequency around $f_{c}=1.75 \mathrm{~Hz}$. Note that this can be considered a best-case interferometric signal, and in general the cw signals were more distorted. It should also be noted that experimental investigations into the step response of the laser PZT revealed a tolerably linear scan movement, and certainly was not the chief contributor to $\mathrm{cw}$ signal distortion. The quadratic polynomial camera response changes the shape of the cw signal (obtuse crests and
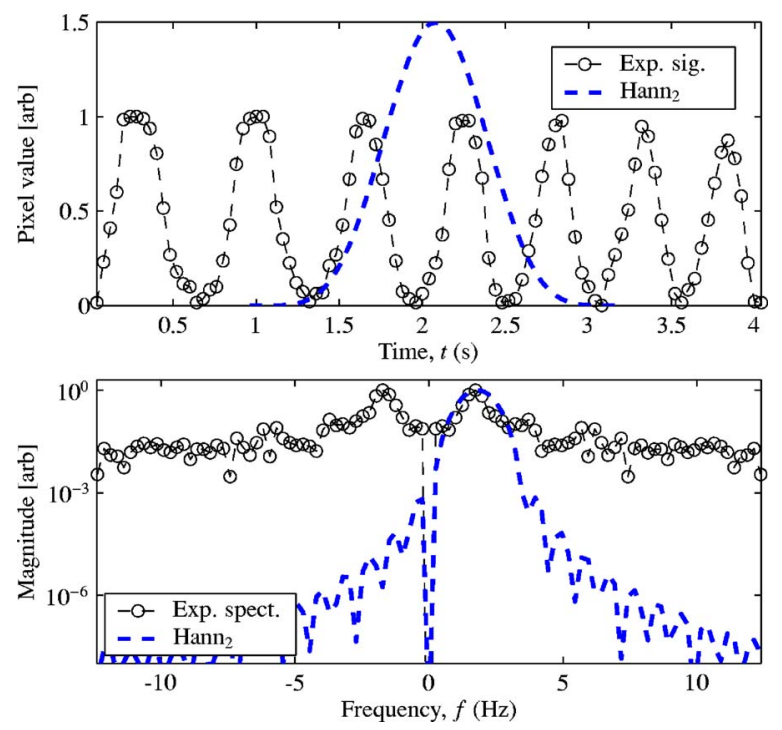

Fig. 4 Normalized experimental interferometric cw signal and $\mathrm{Hann}_{2}$ window with spectra (dc component removed). 
pointy troughs), but this effect would appear repeatable and predictable in time. Thus, experimental cw signal distortion is explained by the wavelength-tuning nonlinearities, evident in Fig. 1. These are attributed to the physical characteristics of the external cavity diode laser, which are dependent on laboratory temperature, operating current, and central wavelength.

By retrieving the phase at a reference position of mirror M1 and tilting the mirror and retrieving that phase, the difference between the two phase measurements corresponds to the mirror tilt. By tilting mirror M1 three times by arbitrary amounts, the full-field tilt measurement is shown in Fig. 5, where each planar tilt is evident. On the $61 \times 34$ pixels, convolution filtering and division operations were clocked at 1.188 and $0.234 \mathrm{~s}$, respectively. In the division process of the phase retrieval algorithm, the reference pixel index was the same for each tilt measurement. Investigations into the mirror surface revealed a flatness within $10 \mathrm{~nm}$. A rough estimation of error in the interferometer was calculated by subtracting two measurements at the same mirror tilt taken in rapid succession. From more than 18 differential measurements (taken in less than

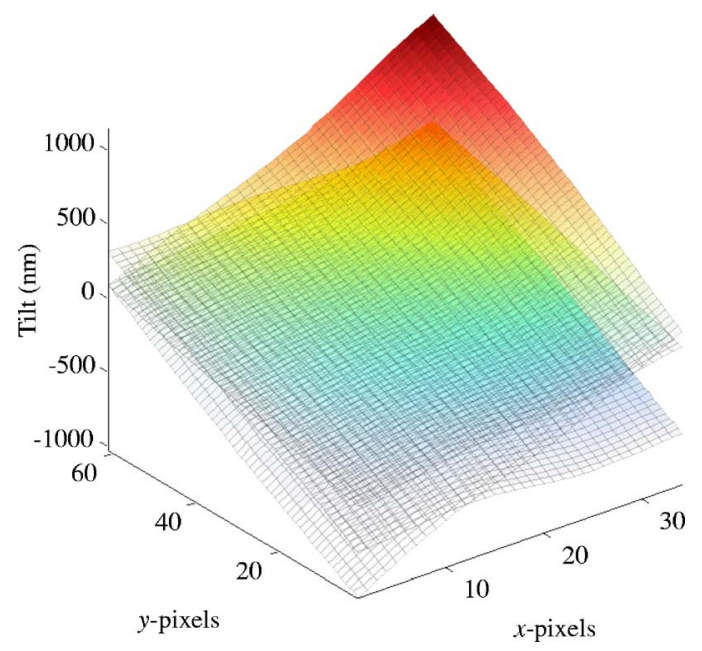

Fig. 5 Phase measurement of mirror tilting at three different inclinations.
$120 \mathrm{~s}$ ), the mean phase error was estimated at 1.863 $\times 10^{-2}$ rad or $4.61 \mathrm{~nm}$, with a standard deviation of 6.949 $\times 10^{-3} \mathrm{rad}$ or $1.72 \mathrm{~nm}$. Note that this approximation is crude: it attempts to encompass all interferometer error (including thermal drifts and vibrations), thus the estimated error is not due entirely to camera and laser nonlinearities.

To conclude, proof-of-principle full-field phase measurement at $\mathrm{C}$-band wavelengths has been demonstrated through wavelength-tuning interferometry. The technique employed a $1555 \mathrm{~nm}$ external cavity diode laser and an inexpensive phosphor-coated IR-sensitive CCD camera, with no moving parts in a Michelson interferometer. In spite of wavelength-tuning nonlinearities, an analytic signal phase retrieval algorithm achieved an accuracy inside $7 \mathrm{~nm}$. However, camera nonlinearity and short response range impose significant drawbacks that may prevent practical future application.

\section{Acknowledgments}

This project was supported by the University of Limerick Foundation and an Enterprise Ireland International Collaboration grant. The work was undertaken within the framework of a Collaboration Agreement (number 186972001-11 SOSC ISP IE) between the University of Limerick, Ireland, and the Institute for Health and Consumer Protection, European Commission Directorate General Joint Research Centre, Italy.

\section{References}

1. I. Yamaguchi, A. Yamamoto, and M. Yano, "Surface topography by wavelength scanning interferometry," Opt. Eng. 39(1), 40-46 (2000).

2. P. de Groot, Appl. Opt. 39, 2658-2663 (2000).

3. L. L. Deck, Appl. Opt. 42, 2354-2365 (2003).

4. M. J. Connelly, Semiconductor Optical Amplifiers, 1st ed., Kluwer Academic Press, Boston, MA (2202).

5. J. K. Sinha and H. V. Tippur, "Infrared interferometry for rough surface measurements application to failure charaterization and flow detection," Opt. Eng. 36, 2233-2239 (1997).

6. T. van Kessel and H. K. Wickramasinghe, Opt. Lett. 24, 1702-1704 (1999).

7. T. L. Schmitz, A. Davies, C. J. Evans, and R. E. Parks, "Silicon wafer thickness variation measurements using the National Institute of Standards and Technology infrared interferometer," Opt. Eng. 42(8), 2281-2290 (2003).

8. M. Takeda, H. Ina, and S. Kobayashi, J. Opt. Soc. Am. 72, 156-160 (1982).

9. M. Suematsu and M. Takeda, Appl. Opt. 30, 4046-4055 (1991).

10. P. Egan, M. J. Connelly, F. Lakestani, and M. P. Whelan, Opt. Lett. 31, 912-914 (2006). 